\title{
DISCUSSION (Dolginov)
}

STEPIEK: My question concerns the problem of the energy source for poloidal currents. If we assume a stationary situation, in the sense that the magnetic field will only decay very slowly, then we need a constant source of energy to support the poloidal currents. But, suppose that the poloidal currents decay faster than the internal currents, leading to an unstable field configuration. Then a kind of kink instability develops and a new stable configuration of the field is reached spontaneously with the poloidal currents having energy drawn from the field itself. Such an instability may occur many times during the star's lifetime. Is this picture possible?

DOLGINOV: For the fast rotating stars the rotation itself is sufficient to maintain the poloidal current. For the slow rotators the 'battery' current, produced by the helium spots, may be sufficient to compensate the energy losses of the stellar current system. As to the origin of the spotted surface, I have not discussed this today, but some ideas about this problem are given in a poster paper which I am presenting at this colloquium. Briefly, it is due to an instability not connected directly with the magnetic field.

ALECIAN: Is it possible that meridional circulation or mass loss bring toward the surface magnetic fields deep in the interior, produced by dynamo effects in the core?

DOLGINOV: No. Meridional circulation can not bring the large-scale dipole magnetic field toward the surface, but it is possible for the small-scale field. The circulation may be strongly suppressed by the effect of the molecular weight redistribution.

KRAUSE: It is true that a magnetic field which is excited in the convective core of a star would take far too long to come to the surface through the radiative zone, if field diffusion is the only thing we take into account. Instabilities, however, provide for a much quicker transport of the field to the surface. The most important one is the buoyancy instability. The density inside a magnetic tube is less than that in the surrounding material, because of the balance of the magnetic pressure. For a certain critical field strength the magnetic tubes become buoyant and $r$ ise to the surface. Sunspots are an excellent example. So, the magnetic field, combined with gravity, produces itself the motion which brings it very quickly to the surface. It is a possibility.

DOLGINOV: Yes, there exists a possibility that the magnetic field may be lifted by buoyancy from the inner core, but the magnetic field rising from the core toward the surface must be accompanied by the transportation of hot matter because the field is frozen in the plasma. The rate of transportation must be sufficiently high because of the field dissipation on the surface where the conductivity is low. Existing models of A stars do not include the possibility of such heat transfer. As far as I am aware, the theory of the lifting of the magnetic field from the inner core has not been elaborated quantitatively.

There is a second problem for the dynamo theory. Why do not all A stars, with convective cores and rotating faster than Ap stars, have

23

C. R. Cowley et al (eds.), Upper Main Sequence Stars with Anomalous Abundances, 23-24.

(C) 1986 by D. Reidel Publishing Company. 
magnetic fields?

DROBYSHEVSKI: I would like to present a somewhat different approach to the origin of magnetic fields observed on CP stars, which exploits the semi-dynamo concept, i.e., MHD magnetic field generation process without self-excitation (see E. M. Drobyshevski, E. N. Kolesnikova \& V. S. Yuferev, Geophys. Astrophys. Fluid Dyn., 23, p. 103, 1983). It is rather difficult to believe that no stars have relic fields. The most probable candidates to have such a field are He-stars and Si Ap stars. They are found in the youngest clusters.

The origin and many inherent peculiarities of the magnetic field in other CP stars may be understood and explained if one assumes a close binary nature and magnetic field generation in situ. Indeed, the duplicity of Am stars is well documented. The magnetic field may be generated by intensive convection concentrated in a very thin outer envelope of the A-star with $M_{*} \approx 1.5-2.0 \mathrm{M}_{\odot}$. If the secondary component is of solar type, say, then due to the continuous accretion of (nonmagnetized) stellar wind, the magnetic field generated in the outer layers will be carried into the interior of the A star and accumulated there. Thus, a first-generation magnetic star forms. It has a weak magnetic field (of order 10 - 100 gauss: see E. M. Drobyshevski \& E. V. Ergma, Astron. Zh., 53, p. 1338, 1976).

The second-generation magnetic star, an intermediate or late type Ap star, is formed when the first-generation magnetic star becomes a red giant and overflows its critical Roche lobe. Then its magnetic field is transported, together with the overflowing matter, on to the secondary component and is strongly amplified by winding on the secondary. Eventually the secondary becomes a magnetic star with a highly disordered but strong magnetic field, and the original primary becomes a distant and virtually undetectable white dwarf. As an example of such a second-generation magnetic star one might consider $\alpha^{2}$ CVn. The causes of chemical peculiarities may also be understood from the same model of a binary origin, and will be discussed in my paper tomorrow.

DOLGINOV: This seems qualitatively possible, but there are definitely single Ap stars, which can not be explained with such a picture. There are no known Ap stars in close binaries with mass exchange. The Am stars are binaries, but again, mass exchange is not observed. In any case it is not easy to get global large-scale dipole fields in the accretion process.

DWORETSKY: The observational detection of small-amplitude, long-period radial velocity variations is very difficult, especially when the star in question has intrinsic variations due to its spottedness. Drobyshevski is referring to systems in which mass exchange has already taken place, and usually the result of this is that the two components end up very much further apart than they began. Drobyshevski's model is not inconsistent with the radial velocity observations. 